\title{
Legendre Wavelet Operational Matrix Method for Solution of Riccati Differential Equation
}

\author{
S. Balaji \\ Department of Mathematics, SASTRA University, Thanjavur 613 401, India \\ Correspondence should be addressed to S. Balaji; balaji_maths@yahoo.com
}

Received 12 February 2014; Revised 20 May 2014; Accepted 5 June 2014; Published 24 June 2014

Academic Editor: Petru Jebelean

Copyright (C) 2014 S. Balaji. This is an open access article distributed under the Creative Commons Attribution License, which permits unrestricted use, distribution, and reproduction in any medium, provided the original work is properly cited.

\begin{abstract}
A Legendre wavelet operational matrix method (LWM) is presented for the solution of nonlinear fractional-order Riccati differential equations, having variety of applications in quantum chemistry and quantum mechanics. The fractional-order Riccati differential equations converted into a system of algebraic equations using Legendre wavelet operational matrix. Solutions given by the proposed scheme are more accurate and reliable and they are compared with recently developed numerical, analytical, and stochastic approaches. Comparison shows that the proposed LWM approach has a greater performance and less computational effort for getting accurate solutions. Further existence and uniqueness of the proposed problem are given and moreover the condition of convergence is verified.
\end{abstract}

\section{Introduction}

In recent years, use of fractional-order derivative goes very strongly in engineering and life sciences and also in other areas of science. One of the best advantages of use of fractional differential equation is modeling and control of many dynamic systems. Fractional-order derivatives are used in fruitful way to model many remarkable developments in those areas of science such as quantum chemistry, quantum mechanics, damping laws, rheology, and diffusion processes [1-5] described through the models of fractional differential equations (FDEs). Modeling of a physical phenomenon depends on two parameters such as the time instant and the prior time history; because of this reason, reasonable modeling through fractional calculus was successfully achieved. The abovementioned advantages and applications of FDEs attracted researchers to develop efficient methods to solve FDEs in order to get accurate solutions to such problems and more active research is still going on in those areas. Most of the FDEs are complicated in their structure; hence finding exact solutions for them cannot be simple. Therefore, one can approach the best accurate solution of FDEs through analytical and numerical methods. Designing accurate or best solution to FDEs, many methods are developed in recent years; each method has its own advantages and limitations. This paper aims to solve a FDE called fractional-order Riccati differential equation, one of the important equations in the family of FDEs. The Riccati equations play an important role in engineering and applied science [6], especially in quantum mechanics [7] and quantum chemistry $[8,9]$. Therefore, solutions to the Riccati differential equations are important to scientists and engineers. Solving fractional-order Riccati differential equation, the most significant methods are Adomian decomposition method [10], homotopy perturbation method [11-14], homotopy analysis method [15, 16], Taylor matrix method [17], Haar wavelet method [18], and combination of Laplace, Adomian decomposition, and Padé approximation [19] methods.

Several numerical methods for approximating the solution of nonlinear fractional-order Riccati differential equations are known. Raja et al. [20] developed a stochastic technique based on particle swarm optimization and simulated annealing. They were used as a tool for rapid global search method and simulated annealing for efficient local search method. A fractional variational iteration method described in the Riemann-Liouville derivative has been applied in [21], to give an analytical approximate solution to nonlinear fractional Riccati differential equation. A combination of finite 
difference method and Padé-variational iteration numerical scheme was proposed by Sweilam et al. [22]. Moreover, an analytical scheme comprising the Laplace transform, the Adomian decomposition method (ADM), and the Padé approximation is given in [19].

However, the abovementioned methods have some restrictions and disadvantages in their performance. For example, very complicated and toughest Adomian polynomials are constructed in the Adomian decomposition method. In the variational iteration method identification Lagrange multiplier yields an underlying accuracy. The homotopy perturbation method needs a linear functional equation in each iteration to solve nonlinear equations; forming these functional equations is very difficult. The performance of the homotopy analysis method very much depends on the choice of the auxiliary parameter $h$ of the zero-order deformation equation. Moreover, the convergence region and implementation of these results are very small.

In recent years, wavelets theory is one of the growing and predominantly new methods in the area of mathematical and engineering research. It has been applied in vast range of engineering sciences; particularly, they are used very successfully for waveform representation and segmentations in signal analysis and time-frequency analysis and in the mathematical sciences it is used in thriving manner for solving variety of linear and nonlinear differential and partial differential equations and fast algorithms for easy implementation [23]. Moreover, wavelets build a connection with fast numerical algorithms [24]; this is due to the fact that wavelets admit the exact representation of a variety of functions and operators. The application of Legendre wavelet and its operational matrix for solving differential, integral, and fractionalorder differential equations is thoroughly considered in $[25,26]$.

In this work, the nonlinear Riccati differential equations of fractional-order are approached analytically by using Legendre wavelets method. The operational matrix of Legendre wavelet is generalized for fractional calculus in order to solve fractional and classical Riccati differential equations. The Legendre wavelet method (LWM) is illustrated by application, and obtained results are compared with recently proposed method for the fractional-order Riccati differential equation. We have adopted Legendre wavelet method to solve Riccati differential equations not only due to its emerging application but also due to its greater convergence region.

The rest of the paper is as follows. In Section 2, definitions and mathematical preliminaries of fractional calculus are presented. In Section 3, Legendre wavelet, its properties, function approximations, and generalized Legendre wavelet operational matrix fractional calculus are discussed. Section 4 establishes application of proposed method in the solution of Riccati differential equations, existence and uniqueness solution of the proposed problem, and convergence analyses of the proposed approach. Section 5 deals with the illustrative examples and their solutions by the proposed approach. Section 6 ends with our conclusion.

\section{Preliminaries and Notations}

The notations, definitions, and preliminary facts present in this section will be used in forthcoming sections of this work. Several definitions of fractional integrals and derivatives have been proposed after the logical definition given by Liouville. Important and few of these definitions include the RiemannLiouville, the Caputo, the Weyl, the Hadamard, the Marchaud, the Riesz, the Grunwald-Letnikov, and the ErdelyiKober. As stated in [25], the Caputo fractional derivative uses initial and boundary conditions of integer order derivatives having some physical interpretations. Because of this specific reason, in this work, we will use the Caputo fractional derivative $D^{\alpha}$ proposed by Caputo [27] in the theory of viscoelasticity.

The Caputo fractional derivative of order $\alpha>0(\alpha \epsilon$ $R, n-1<\alpha \leq n, n \in N)$ and $h:(0, \infty) \rightarrow R$ is continuous and is defined by

$$
D^{\alpha} f(t)=I^{n-\alpha}\left(\frac{d^{n}}{d t^{n}} f(t)\right)
$$

where

$$
I^{\alpha} f(t)=\frac{1}{\Gamma(\alpha)} \int_{0}^{t}(t-s)^{\alpha-1} f(s) d s
$$

is the Riemann-Liouville fractional integral operator of order $\alpha>0$ and $\Gamma$ is the gamma function.

The fractional integral of $t^{\beta}, \beta>-1$ is given as

$$
I^{\alpha}(t-\alpha)^{\beta}=\frac{\Gamma(\beta+1)}{\Gamma(\beta+\alpha+1)}(t-a)^{\beta+\alpha}, \quad a \geq 0 .
$$

Properties of fractional integrals and derivatives are as follows [28], for $\alpha, \beta>0$.

The fractional-order integral satisfies the semigroup property

$$
I^{\alpha}\left(I^{\beta} f(t)\right)=I^{\beta}\left(I^{\alpha} f(t)\right)=I^{\alpha+\beta} f(t) .
$$

The integer order derivative $D^{n}$ and fractional-order derivative $D^{\alpha}$ commute with each other:

$$
D^{n}\left(D^{\alpha} f(t)\right)=D^{\alpha}\left(D^{n} f(t)\right)=D^{n+\alpha} f(t) .
$$

The fractional integral operator and fractional derivative operator do not satisfy the commutative property. In general,

$$
I^{\alpha}\left(D^{\alpha} f(t)\right)=f(t)-\sum_{k=0}^{n-1} f^{(k)}(0) \frac{t^{k}}{k !} .
$$

But in the reverse way we have

$$
D^{\alpha}\left(I^{\beta} f(t)\right)=D^{\alpha-\beta} f(t) .
$$




\section{Generalized Legendre Wavelet Operational Matrix to Fractional Integration}

3.1. Legendre Wavelets. A family of functions were constituted by wavelets and constructed from dilation and translation of a single function called mother wavelet. When the parameters $a$ of dilation and $b$ of translation vary continuously, following are the family of continuous wavelets [29]:

$$
\psi_{a, b}(t)=|a|^{-1 / 2} \psi\left(\frac{t-b}{a}\right), \quad a, b \in \mathfrak{R}, a \neq 0 .
$$

If the parameters $a$ and $b$ are restricted to discrete values as $a=a_{0}^{-k}, b=n b_{0} a_{0}^{-k}, a_{0}>1, b_{0}>0$, and $n$ and $k$ are positive integers, following are the family of discrete wavelets:

$$
\psi_{k, n}(t)=\left|a_{0}\right|^{k / 2} \psi\left(a_{0}^{k} t-n b_{0}\right),
$$

where $\psi_{k, n}(t)$ form a wavelet basis for $L^{2}(R)$. In particular, when $a_{0}=2$ and $b_{0}=1, \psi_{k, n}(t)$ form an orthonormal basis [29].

Legendre wavelets $\psi_{n, m}(t)=\psi(k, \widehat{n}, m, t)$ have four arguments; $\widehat{n}=2 n-1, n=1,2,3, \ldots, 2^{k-1}, k$ can assume any positive integer, $m$ is the order for Legendre polynomials, and $t$ is the normalized time. They are defined on the interval $[0,1)$ as $[30,31]$

$$
\begin{aligned}
& \psi_{n m}(t) \\
& \quad= \begin{cases}\sqrt{m+\frac{1}{2}} 2^{k / 2} P_{m}\left(2^{k} t-\widehat{n}\right), & \text { for } \frac{\widehat{n}-1}{2^{k}} \leq t<\frac{\widehat{n}+1}{2^{k}}, \\
0, & \text { otherwise, }\end{cases}
\end{aligned}
$$

where $m=0,1, \ldots, M-1$ and $n=1,2,3, \ldots, 2^{k-1}$. The coefficient $\sqrt{m+(1 / 2)}$ is for orthonormality, the dilation parameter is $a=2^{-k}$, and translation parameter is $b=$ $\widehat{n} 2^{-k} . P_{m}(t)$ are the well-known Legendre polynomials of order $m$ defined on the interval $[-1,1]$ and can be determined with the aid of the following recurrence formulae:

$$
\begin{gathered}
P_{0}(t)=1, \quad P_{1}(t)=t, \\
P_{m+1}(t)=\left(\frac{2 m+1}{m+1}\right) t P_{m}(t)-\left(\frac{m}{m+1}\right) P_{m-1}(t), \\
m=1,2,3, \ldots .
\end{gathered}
$$

The Legendre wavelet series representation of the function $f(t)$ defined over $[0,1)$ is given by

$$
f(t)=\sum_{n=1}^{\infty} \sum_{m=0}^{\infty} c_{n m} \psi_{n m}(t)
$$

where $c_{n m}=\left\langle f(t), \psi_{n m}(t)\right\rangle$, in which $\langle\cdot, \cdot\rangle$ denotes the inner product. If the infinite series in (12) is truncated, then (12) can be written as

$$
f(t) \cong \sum_{n=1}^{2^{k-1}} \sum_{m=0}^{M-1} c_{n m} \psi_{n m}(t)=C^{T} \Psi(t)=\widehat{f}(t),
$$

where $C$ and $\Psi(t)$ are $2^{k-1} M \times 1$ matrices given by

$$
\begin{gathered}
C=\left[c_{10}, c_{11}, \ldots, c_{1 M-1}, c_{20}, c_{21}, \ldots, c_{2 M-1},\right. \\
\left.\ldots, c_{2^{k-1} 0}, c_{2^{k-1} 1}, \ldots, c_{2^{k-1} M-1},\right]^{T} . \\
\Psi(t)=\left[\psi_{10}(t), \psi_{11}(t), \ldots, \psi_{1 M-1}(t), \psi_{20}(t),\right. \\
\psi_{21}(t), \ldots, \psi_{2 M-1}(t), \ldots, \\
\left.\psi_{2^{k-1} 0}(t), \psi_{2^{k-1} 1}(t), \ldots, \psi_{2^{k-1} M-1}(t),\right]^{T} .
\end{gathered}
$$

Taking suitable collocation points as

$$
t_{i}=\cos \left(\frac{(2 i+1) \pi}{2^{k} M}\right) \quad i=1,2, \ldots, 2^{k-1} M,
$$

we defined the $\widehat{m}$-square Legendre matrix

$$
\begin{array}{r}
\phi_{\widehat{m} \times \widehat{m}}=\left[\Psi\left(\cos \left(\frac{3 \pi}{2^{k} M}\right)\right) \Psi\left(\cos \left(\frac{5 \pi}{2^{k} M}\right)\right)\right. \\
\left.\ldots \Psi\left(\cos \left(\frac{\left(2^{k} M+1\right) \pi}{2^{k} M}\right)\right)\right],
\end{array}
$$

where $\widehat{m}=2^{k-1} M$; correspondingly, we have

$$
\begin{aligned}
\widehat{f}=\left[\widehat{f}\left(\cos \left(\frac{3 \pi}{2^{k} M}\right)\right)\right. \\
\left.\quad \times \widehat{f}\left(\cos \left(\frac{5 \pi}{2^{k} M}\right)\right) \cdots \widehat{f}\left(\cos \left(\frac{\left(2^{k} M+1\right) \pi}{2^{k} M}\right)\right)\right] \\
=C^{T} \phi_{\widehat{m} \times \widehat{m}} .
\end{aligned}
$$

The Legendre matrix $\phi_{\widehat{m} \times \widehat{m}}$ is an invertible matrix, and the coefficient vector $C^{T}$ is obtained by $C^{T}=\widehat{f} \phi_{\widehat{m} \times \widehat{m}}^{-1}$.

3.2. Operational Matrix of the Fractional Integration. The integration of the $\Psi(t)$ defined in (14) can be approximated by Legendre wavelet series with Legendre wavelet coefficient matrix $P$ :

$$
\int_{0}^{t} \Psi(t) d t=P_{\widehat{m} \times \widehat{m}} \Psi(t)
$$

where the $\widehat{m}$-square matrix $P$ is called Legendre wavelet operational matrix of integration.

The $m$-set of block-pulse functions is defined on $[0,1)$ as follows:

$$
b_{i}(t)=\left\{\begin{array}{l}
1, \quad \frac{i}{m} \leq t<\frac{(i+1)}{m} \\
0, \quad \text { otherwise }
\end{array}\right.
$$

where $i=0,1,2,3, \ldots m$. 
The functions $b_{i}$ are disjoint and orthogonal. That is,

$$
\begin{gathered}
b_{i}(t) b_{j}(t)= \begin{cases}b_{i}(t), & i=j, \\
0, & i \neq j,\end{cases} \\
\int_{0}^{1} b_{i}(t) b_{j}(t) d t= \begin{cases}\frac{1}{m}, & i=j, \\
0, & i \neq j .\end{cases}
\end{gathered}
$$

The orthogonality property of block-pulse function is obtained from the disjointness property.

An arbitrary function $f \in L_{2}[0,1)$ can be expanded into block-pulse functions as

$$
f(t) \approx \sum_{i=0}^{m-1} f_{i} b_{i}(t)=f^{T} B(t)
$$

where $f_{i}$ are the coefficients of the block-pulse function, given by

$$
f_{i}=\frac{m}{l} \int_{0}^{l} f(t) b_{i}(t)
$$

The Legendre wavelets can be expanded into $m$-set of blockpulse functions as

$$
\Psi(t)=\phi_{\widehat{m} \times \widehat{m}} B(t),
$$

where $B(t)=\left[b_{0}(t) b_{1}(t) \cdots b_{i}(t) \cdots b_{m-1}(t)\right]^{T}$.
The fractional integral of block-pulse function vector can be written as

$$
\left(I^{\alpha} B\right)(t)=F_{m \times m}^{\alpha} B(t),
$$

where $F_{m \times m}^{\alpha}$ is given in [32].

Now, we introduce the derivation process of the Legendre wavelet operational matrix of the fractional integration:

$$
\left(I^{\alpha} \Psi\right)(t) \approx P_{\widehat{m} \times \widehat{m}}^{\alpha} \Psi(t)
$$

where the $\widehat{m}$-square matrix $P_{\widehat{m} \times \widehat{m}}^{\alpha}$ is called Legendre wavelet operational matrix of the fractional integration.

Using (23) and (25), we have

$$
\begin{aligned}
\left(I^{\alpha} \Psi\right)(t) & \approx\left(I^{\alpha} \phi_{\widehat{m} \times \widehat{m}} B\right)(t)=\phi_{\widehat{m} \times \widehat{m}}\left(I^{\alpha} B\right)(t) \\
& \approx \phi_{\widehat{m} \times \widehat{m}}\left(F^{\alpha} B\right)(t) .
\end{aligned}
$$

From (25) and (26), we get

$$
P_{\widehat{m} \times \widehat{m}}^{\alpha} \Psi(t)=\phi_{\widehat{m} \times \widehat{m}} F^{\alpha} B(t),
$$

and by (23), (27) becomes

$$
P_{\widehat{m} \times \widehat{m}}^{\alpha} \phi_{\widehat{m} \times \widehat{m}} B(t)=\phi_{\widehat{m} \times \widehat{m}} F^{\alpha} B(t) .
$$

Then, the Legendre wavelet operational matrix $P_{\widehat{m} \times \widehat{m}}^{\alpha}$ of fractional integration is given by

$$
P_{\widehat{m} \times \widehat{m}}^{\alpha}=\phi_{\widehat{m} \times \widehat{m}} F^{\alpha} \phi_{\widehat{m} \times \widehat{m}}^{-1} .
$$

Following is the Legendre wavelet operational matrix $P_{\widehat{m} \times \widehat{m}}^{\alpha}$ of fractional-order integration, for the particular values of $k=2$, $M=3$, and $\alpha=0.5$ :

$$
P_{6 \times 6}^{0.5}=\left(\begin{array}{cccccc}
0.5415 & 0.4324 & 0.1819 & -0.0871 & -0.0179 & 0.0154 \\
0 & 0.5415 & 0 & 0.1819 & 0 & -0.0179 \\
-0.2046 & 0.071 & 0.2243 & -0.0449 & 0.0798 & 0.0119 \\
0 & -0.2046 & 0 & 0.2243 & 0 & 0.0798 \\
0.1781 & 0.2506 & -0.0252 & -0.0652 & 0.1555 & 0.0143 \\
0 & 0.1781 & 0 & -0.0252 & 0 & 0.1555
\end{array}\right)
$$

\section{Application to Fractional Riccati Differential Equation}

In this section, we use the generalized Legendre wavelet operational matrix to solve nonlinear Riccati differential equation and we discuss the existence and uniqueness of solutions with initial conditions and convergence criteria of the proposed LWM approach.

Consider the fractional-order Riccati differential equation of the form

$$
\begin{array}{r}
D^{\alpha} y(t)=P(t) y^{2}+Q(t) y+R(t), \\
t>0, \quad 0<\alpha \leq 1,
\end{array}
$$

subject to the initial condition

$$
y(0)=k
$$

Let us suppose that the functions $D^{\alpha} y(t), P(t), Q(t)$, and $R(t)$ are approximated using Legendre wavelet as follows:

$$
\begin{array}{ll}
D^{\alpha} y(t)=U^{T} \Psi(t), & P(t)=V^{T} \Psi(t), \\
Q(t)=W^{T} \Psi(t), & R(t)=X^{T} \Psi(t),
\end{array}
$$

where $U, V, W, X$, and $\Psi(t)$ are given in (14).

Using (6), we can write

$$
y(t)=I^{\alpha}\left(D^{\alpha} y(t)\right)-y(0) .
$$


By (25) and (32), (34) leads to

$$
y(t) \approx U^{T} P^{\alpha} \Psi(t)+Y_{0}^{T} \Psi(t)=C^{T} \Psi(t),
$$

where $y(0)=k \approx Y_{0}^{T} \Psi(t), \quad C=\left(U^{T} P_{\widehat{m} \times \widehat{m}}^{\alpha}+Y_{0}^{T}\right)^{T}$.

Substituting (33) and (35) into (31), we have

$$
\begin{aligned}
U^{T} \Psi(t)= & V^{T} \Psi(t)\left[C^{T} \Psi(t)\right]^{2} \\
& +W^{T} \Psi(t) C^{T} \Psi(t)+X^{T} \Psi(t) .
\end{aligned}
$$

Substituting (23) into (36), we have

$$
U^{T} \phi_{\widehat{m} \times \widehat{m}}=V^{T}\left[C^{T} \phi_{\widehat{m} \times \widehat{m}}\right]^{2}+W^{T} C^{T} \phi_{\widehat{m} \times \widehat{m}}+X^{T},
$$

where $C, V, W$, and $\phi_{\widehat{m} \times \widehat{m}}$ are known. Equation (37) represents a system of nonlinear equations with unknown vector $U$. This system of nonlinear equations can be solved by Newton method for the unknown vector $U$ and we can get the approximation solution by including $U$ into (35).

4.1. Existence and Uniqueness of Solutions. Consider the fractional-order Riccati differential equation of the forms (31) and (32). The nonlinear term in (31) is $y^{2}$ and $P(t), Q(t)$, and $R(t)$ are known functions. For $\alpha=1$, the fractional-order Riccati converts into the classical Riccati differential equation.

Definition 1. Let $I=[0, l], l<\infty$, and $C(I)$ be the class of all continuous functions defined on $I$, with the norm

$$
\|y\|=\sup _{t \in I}\left|e^{-h t} y(t)\right|, \quad h>0,
$$

which is equivalent to the sup norm of $y$. That is, $\|y\|=$ $\sup _{t \in I}\left|e^{-h t} y(t)\right|$.

Remark. Assume that solution $y(t)$ of fractional-order Riccati differential equations (31) and (32) belongs to the space $S=$ $\{y \in R:|y| \leq c, c$ is any constant $\}$, in order to study the existence and uniqueness of the initial value problem.

Definition 2. The space of integrable functions $L_{1}[0, l]$ in the interval $[0, l]$ is defined as

$$
L_{1}[0, l]=\left\{u(t): \int_{0}^{l}|u(t)| d t<\infty\right\} .
$$

Theorem 3. The initial value problem given by (31) and (32) has a unique solution:

$$
y \in C(I), \quad y^{\prime} \in X=\left\{y \in L_{1}[0, l],\|y\|=\left\|e^{-h t} y(t)\right\|_{L_{1}}\right\} .
$$

Proof. By (1), the fractional differential equation (31) can be written as

$$
I^{1-\alpha} \frac{d y(t)}{d t}=P(t) y^{2}+Q(t) y+R(t)
$$

and becomes

$$
y(t)=I^{\alpha}\left(P(t) y^{2}+Q(t) y+R(t)\right) .
$$

Now we define the operator $\Theta: C(I) \rightarrow C(I)$ by

$$
\Theta y(t)=I^{\alpha}\left(P(t) y^{2}+Q(t) y+R(t)\right),
$$

and then

$$
\begin{gathered}
e^{-h t}(\Theta y-\Theta w) \\
=e^{-h t} I^{\alpha}\left[\left(P y^{2}(t)+Q y(t)+R\right)\right. \\
\left.-\left(P w^{2}(t)+Q w(t)+R\right)\right] \\
\leq \frac{1}{\Gamma(\alpha)} \int_{0}^{t}(t-s)^{\alpha-1} e^{-h(t-s)} \\
\times[(y(s)-w(s))(y(s)+w(s)) \\
-k(y(s)-w(s))] e^{-h s} d s \\
\leq\|y-w\| \frac{1}{\Gamma(\alpha)} \int_{0}^{t} s^{\alpha-1} e^{-h s} d s ;
\end{gathered}
$$

hence, we have

$$
\|\Theta y-\Theta w\|<\|y-w\|,
$$

which implies that the operator given by (43) has a unique fixed point and consequently the given integral equation has a unique solution $y(t) \in C(I)$. Also we can see that

$$
\left.I^{\alpha}\left(P(t) y^{2}+Q(t) y+R(t)\right)\right|_{t=0}=k .
$$

Now, from (42), we have

$$
\begin{aligned}
y(t)= & {\left[\frac{t^{\alpha}}{\Gamma(\alpha+1)}\left(P y_{0}^{2}+Q y_{0}+R\right)\right.} \\
& \left.+I^{\alpha+1}\left(P^{\prime} y^{2}+2 y^{\prime} P+Q^{\prime} y+Q y^{\prime}+R^{\prime}\right)\right], \\
\frac{d y}{d t}=[ & \frac{t^{\alpha-1}}{\Gamma(\alpha)}\left(P y_{0}^{2}+Q y_{0}+R\right) \\
& \left.+I^{\alpha}\left(P^{\prime} y^{2}+2 y^{\prime} P+Q^{\prime} y+Q y^{\prime}+R^{\prime}\right)\right], \\
e^{-h t} y^{\prime}(t)= & e^{-h t}\left[\frac{t^{\alpha-1}}{\Gamma(\alpha)}\left(P y_{0}^{2}+Q y_{0}+R\right)\right. \\
& \left.+I^{\alpha}\left(P^{\prime} y^{2}+2 y^{\prime} P+Q^{\prime} y+Q y^{\prime}+R^{\prime}\right)\right],
\end{aligned}
$$

from which we can deduce that $y^{\prime}(t) \in C(I)$ and $y^{\prime}(t) \in S$. 
Now, again from (42), (43), and (46), we get

$$
\begin{aligned}
& \frac{d y}{d t}=\frac{d}{d t} I^{\alpha}\left[P y^{2}(t)+Q y(t)+R\right], \\
& I^{1-\alpha} \frac{d y}{d t}=I^{1-\alpha} \frac{d}{d t} I^{\alpha}\left[P y^{2}(t)+Q y(t)+R\right] \\
& =\frac{d}{d t} I^{1-\alpha} I^{\alpha}\left[P y^{2}(t)+Q y(t)+R\right], \\
& D^{\alpha} y(t)=\frac{d}{d t} I\left[P y^{2}(t)+Q y(t)+R\right] \\
& =P y^{2}(t)+Q y(t)+R, \\
& y(0)=\left.I^{\alpha}\left(P y^{2}(t)+Q y(t)+R\right)\right|_{t=0}=k,
\end{aligned}
$$

which implies that the integral equation (46) is equivalent to the initial value problem (32) and the theorem is proved.

\subsection{Convergence Analyses. Let}

$$
\psi_{k, n}(t)=\left|a_{0}\right|^{k / 2} \psi\left(a_{0}^{k} t-n b_{0}\right)
$$

where $\psi_{k, n}(t)$ form a wavelet basis for $L^{2}(R)$. In particular, when $a_{0}=2$ and $b_{0}=1, \psi_{k, n}(t)$ form an orthonormal basis [29].

By (14), let $y(t)=\sum_{i=1}^{M-1} c_{1 i} \psi_{1 i}(t)$ be the solution of (31) where $c_{1 i}=\left\langle y(t), \psi_{1 i}(t)\right\rangle$, for $k=1$ in which $\langle\cdot, \cdot\rangle$ denotes the inner product:

$$
y(t)=\sum_{i=1}^{n}\left\langle y(t), \psi_{1 i}(t)\right\rangle \psi_{1 i}(t)
$$

Let $\beta_{j}=\langle y(t), \psi(t)\rangle$ where $\psi(t)=\psi_{1 i}(t)$.

Let $x_{n}=\sum_{j=1}^{n} \beta_{j} \psi\left(t_{j}\right)$ be a sequence of partial sums. Then,

$$
\begin{aligned}
\left\langle y(t), x_{n}\right\rangle & =\left\langle y(t), \sum_{j=1}^{n} \beta_{j} \psi\left(t_{j}\right)\right\rangle \\
& =\sum_{j=1}^{n} \overline{\beta_{j}}\left\langle y(t), \psi\left(t_{j}\right)\right\rangle \\
& =\sum_{j=1}^{n} \overline{\beta_{j}} \beta_{j} \\
& =\sum_{j=1}^{n}\left|\beta_{j}\right|^{2} .
\end{aligned}
$$

Further,

$$
\begin{aligned}
\left\|x_{n}-x_{m}\right\|^{2} & =\left\|\sum_{j=m+1}^{n} \beta_{j} \psi\left(t_{j}\right)\right\|^{2} \\
& =\left\langle\sum_{i=m+1}^{n} \beta_{i} \psi\left(t_{i}\right), \sum_{j=m+1}^{n} \beta_{j} \psi\left(t_{j}\right)\right\rangle \\
& =\sum_{i=m+1}^{n} \sum_{j=m+1}^{n} \beta_{i} \overline{\beta_{j}}\left\langle\psi\left(t_{i}\right), \psi\left(t_{j}\right)\right\rangle \\
& =\sum_{j=m+1}^{n}\left|\beta_{j}\right|^{2} .
\end{aligned}
$$

As $n \rightarrow \infty$, from Bessel's inequality, we have $\sum_{j=1}^{\infty}\left|\beta_{j}\right|^{2}$ is convergent.

It implies that $\left\{x_{n}\right\}$ is a Cauchy sequence and it converges to $x$ (say).

Also,

$$
\begin{aligned}
\left\langle x-y(t), \psi\left(t_{j}\right)\right\rangle & =\left\langle x, \psi\left(t_{j}\right)\right\rangle-\left\langle y(t), \psi\left(t_{j}\right)\right\rangle \\
& =\left\langle\lim _{n \rightarrow \infty} x_{n}, \psi\left(t_{j}\right)\right\rangle-\beta_{j} \\
& =\lim _{n \rightarrow \infty}\left\langle x_{n}, \psi\left(t_{j}\right)\right\rangle-\beta_{j} \\
& =\lim _{n \rightarrow \infty}\left\langle\sum_{j=1}^{n} \beta_{j} \psi\left(t_{j}\right), \psi\left(t_{j}\right)\right\rangle-\beta_{j} \\
& =\beta_{j}-\beta_{j}=0,
\end{aligned}
$$

which is possible only if $y(t)=x$. That is, both $y(t)$ and $x_{n}$ converge to the same value, which indeed give the guarantee of convergence of LWM.

\section{Numerical Examples}

In order to show the effectiveness of the Legendre wavelets method (LWM), we implement LWM to the nonlinear fractional Riccati differential equations. All the numerical experiments were carried out on a personal computer with some MATLAB codes. The specifications of PC are Intel core i5 processor and with Turbo boost up to $3.1 \mathrm{GHz}$ and $4 \mathrm{~GB}$ of DDR3 memory. The following problems of nonlinear Riccati differential equations are solved with real coefficients.

Example 1. Consider the following nonlinear fractional Riccati differential equation:

$$
D^{\alpha} y(t)=1+2 y(t)-y^{2}(t), \quad 0<\alpha \leq 1,
$$

with initial condition

$$
y(0)=0 \text {. }
$$

Exact solution for $\alpha=1$ was found to be

$$
y(t)=1+\sqrt{2} \tanh \left(\sqrt{2 t}+\frac{1}{2} \log \left(\frac{\sqrt{2}-1}{\sqrt{2}+1}\right)\right) .
$$




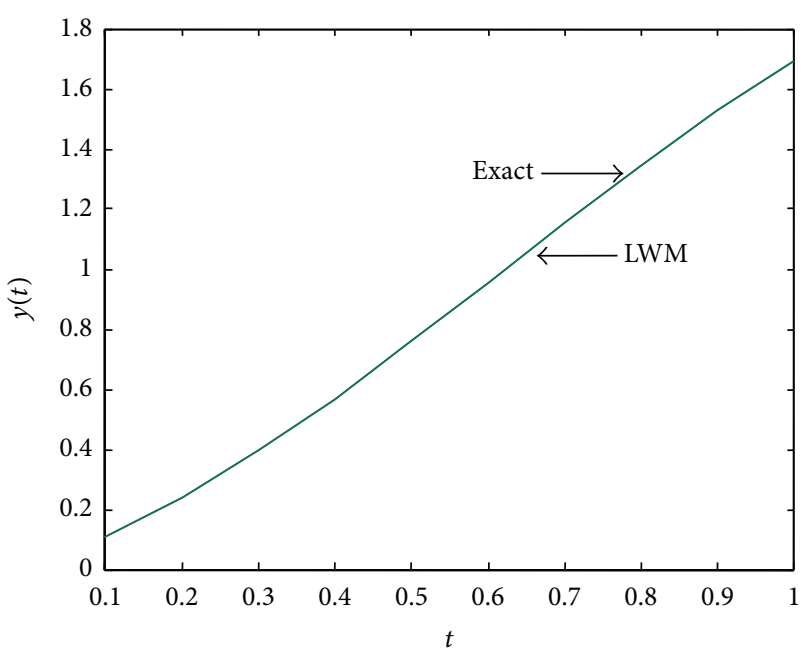

FIgURE 1: Numerical results of Example 1 by LWM for $\alpha=1$.

The integral representation of (54) and (55) is given by

$$
\begin{gathered}
I^{\alpha}\left(D^{\alpha} y(t)\right)=I^{\alpha}\left(1+2 y(t)-y^{2}(t)\right), \\
y(t)=y(0)+\frac{t^{\alpha}}{\Gamma(\alpha+1)}+2 I^{\alpha} y(t)-I^{\alpha} y^{2}(t) .
\end{gathered}
$$

Let

$$
y(t)=C^{T} \Psi(t)
$$

and then

$$
\begin{aligned}
I^{\alpha} y(t) & =C^{T} I^{\alpha} \Psi(t) \\
& =C^{T} P_{2^{k-1} M \times 2^{k-1} M}^{\alpha} \Psi(t) .
\end{aligned}
$$

By substituting (59) and (60) into (58), we get the following system of algebraic equations:

$$
\begin{aligned}
C^{T} \Psi(t)= & \frac{t^{\alpha}}{\Gamma(\alpha+1)}+2 C^{T} P_{2^{k-1} M \times 2^{k-1} M}^{\alpha} \Psi(t) \\
& -C^{T} P_{2^{k-1} M \times 2^{k-1} M}^{2 \alpha} \Psi(t) .
\end{aligned}
$$

By solving the above system of linear equations, we can find the vector $C$. Numerical results are obtained for different values of $k, M$, and $\alpha$. Solution obtained by the proposed LWM approach for $\alpha=1, k=1$, and $M=3$ is given in Figure 1 and for different values of $\alpha=0.6,0.7,0.8$, and 0.9 and for $k=2$ and $M=5$ is graphically given in Figure 2. It can be seen from Figure 1 that the solution obtained by the proposed LWM approach is more close to the exact solution. Table 1 describes the efficiency of the proposed method by comparing with the methods in $[20,22]$ through their absolute error. The following is used for the errors of the approximation $\hat{y}(t)$ of $y(t)$; that is, $\|y-\widehat{y}\|=\max |y(t)-\widehat{y}(t)|$. Table 1 shows that very high accuracies are obtained for $k=3$ and $M=5$ by the present method.

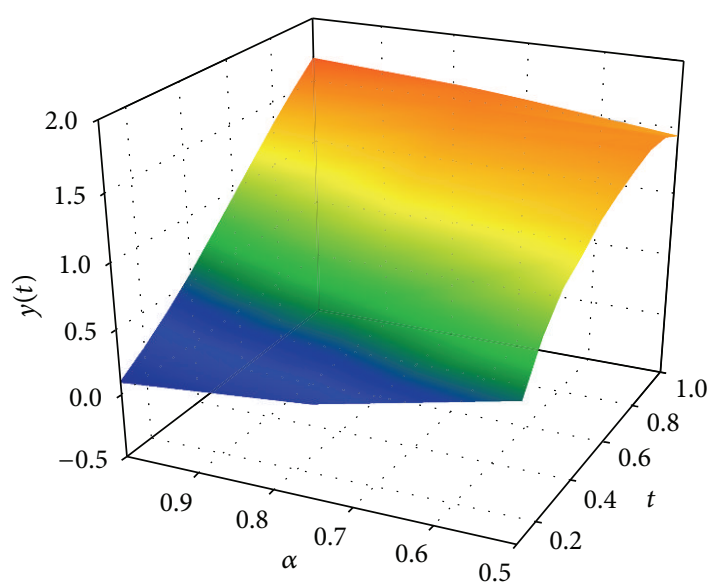

FIgURE 2: Numerical results of Example 1 by LWM for different values of $\alpha$.

Example 2. Consider another fractional-order Riccati differential equation:

$$
D^{\alpha} y(t)=1-y^{2}(t), \quad 0<\alpha \leq 1
$$

with initial condition

$$
y(0)=0
$$

Exact solution for the above equation was found to be

$$
y(t)=\frac{e^{2 t}-1}{e^{2 t}+1}
$$

The integral representation of (62) and (63) is given by

$$
y(t)=y(0)+\frac{t^{\alpha}}{\Gamma(\alpha+1)}-I^{\alpha} y^{2}(t) .
$$

Let

$$
y(t)=C^{T} \Psi(t)
$$

and then

$$
\begin{aligned}
I^{\alpha} y(t) & =C^{T} I^{\alpha} \Psi(t) \\
& =C^{T} P_{2^{k-1} M \times 2^{k-1} M}^{\alpha} \Psi(t) .
\end{aligned}
$$

By substituting (66) and (67) in (62), we get the following system of algebraic equations:

$$
C^{T} \Psi(t)=\frac{t^{\alpha}}{\Gamma(\alpha+1)}-C^{T} P_{2^{k-1} M \times 2^{k-1} M}^{2 \alpha} \Psi(t) .
$$

By solving the above system of linear equations, we can find the vector $C$. Numerical results are obtained for different values of $k, M$, and $\alpha$. Results obtained by LWM for $\alpha=1, k=$ 2 , and $M=3$ are shown in Figure 3 and it can be seen from the figure that solution given by the LWM merely coincides with the exact solution. Figure 4 shows the obtained results of (62) and (63) by LWM for different values of $\alpha$ and for $k=2$ and 
TABLE 1: Numerical results of Example 1 for $\alpha=1$.

\begin{tabular}{lcccccc}
\hline$t$ & Exact solution & Absolute error in [22] & Absolute error in $[20]$ & $\begin{array}{c}\text { LWM } \\
M=2, k=2\end{array}$ & $\begin{array}{c}\text { LWM } \\
M=3, k=2\end{array}$ \\
\hline 0.1 & 0.099667 & $7.51 E-09$ & $9.30 E-05$ & 0 & 0 & 0 \\
0.2 & 0.197375 & $1.52 E-06$ & $2.93 E-02$ & $1.91 E-08$ & 0 \\
0.3 & 0.291312 & $3.93 E-05$ & $3.78 E-03$ & $2.72 E-08$ & $1.45 E-13$ & 0 \\
0.4 & 0.379948 & $4.32 E-04$ & $2.81 E-03$ & $1.65 E-08$ & $1.17 E-13$ & $1.94 E-16$ \\
0.5 & 0.462117 & $8.41 E-04$ & $9.80 E-04$ & $1.31 E-08$ & $3.28 E-13$ & $3.20 E-16$ \\
0.6 & 0.537049 & $2.94 E-05$ & $7.93 E-03$ & $1.98 E-08$ & $4.97 E-13$ & $1.24 E-16$ \\
0.7 & 0.604367 & $3.35 E-04$ & $9.44 E-03$ & $2.52 E-08$ & $6.32 E-13$ & $1.58 E-16$ \\
0.8 & 0.664036 & $5.44 E-04$ & $1.17 E-02$ & $2.94 E-08$ & $7.36 E-13$ & $1.84 E-16$ \\
0.9 & 0.716297 & $6.56 E-09$ & $3.96 E-02$ & $3.23 E-08$ & $8.12 E-13$ & $1.94 E-16$ \\
1.0 & 0.761594 & $2.53 E-06$ & $2.95 E-02$ & $2.63 E-08$ & $4.62 E-13$ & $1.99 E-16$ \\
\hline
\end{tabular}

TABLE 2: Numerical results of Example 2 for $\alpha=1$.

\begin{tabular}{|c|c|c|c|c|c|c|}
\hline$t$ & Exact solution & Absolute error in [22] & Absolute error in [20] & $\begin{array}{c}\text { LWM } \\
M=2, k=2\end{array}$ & $\begin{array}{c}\text { LWM } \\
M=3, k=2\end{array}$ & $\begin{array}{c}\text { LWM } \\
M=5, k=3\end{array}$ \\
\hline 0.1 & 0.110295 & $1.23 E-15$ & $2.81 E-03$ & 0 & 0 & 0 \\
\hline 0.2 & 0.241976 & $5.24 E-15$ & $3.83 E-04$ & 0 & 0 & 0 \\
\hline 0.3 & 0.395104 & $8.16 E-15$ & $1.23 E-04$ & 0 & 0 & 0 \\
\hline 0.4 & 0.567812 & $1.15 E-12$ & $2.86 E-03$ & $1.68 E-12$ & $1.66 E-15$ & $1.43 E-16$ \\
\hline 0.5 & 0.756014 & $6.17 E-12$ & $4.38 E-04$ & $2.22 E-12$ & $2.12 E-15$ & $1.66 E-16$ \\
\hline 0.6 & 0.953566 & $4.55 E-11$ & $5.19 E-02$ & $1.15 E-12$ & $1.10 E-15$ & $1.54 E-16$ \\
\hline 0.7 & 1.152946 & $7.57 E-10$ & $2.14 E-02$ & $1.27 E-12$ & $1.11 E-15$ & $1.33 E-16$ \\
\hline 0.8 & 1.346363 & $6.33 E-09$ & $1.42 E-02$ & $1.87 E-12$ & $2.01 E-15$ & $1.75 E-16$ \\
\hline 0.9 & 1.526911 & $3.67 E-08$ & $6.98 E-03$ & $1.93 E-12$ & $2.66 E-15$ & $1.87 E-16$ \\
\hline 1.0 & 1.689498 & $1.64 E-07$ & $4.96 E-03$ & $1.56 E-12$ & $1.66 E-15$ & $1.64 E-16$ \\
\hline
\end{tabular}

$M=5$. Table 2 describes the efficiency of the proposed method by comparing with the methods in [20,22] through their absolute error. Table 1 shows that very high accuracies are obtained for $k=3$ and $M=5$ by the present method and from these results we can identify that guarantee of convergence of the proposed LWM approach is very high.

Example 3. Let us consider another problem of nonlinear Riccati differential equation:

$$
D^{\alpha} y(t)=t^{2}+y^{2}(t), \quad 0<\alpha \leq 1, t \geq 0
$$

with initial condition

$$
y(0)=1
$$

When $\alpha=1$, its exact solution is given by

$$
y(t)=\frac{t\left(J_{-3 / 4}\left(t^{2} / 2\right) \Gamma(1 / 4)+2 J_{3 / 4}\left(t^{2} / 2\right) \Gamma(3 / 4)\right)}{J_{1 / 4}\left(t^{2} / 2\right) \Gamma(1 / 4)-2 J_{-1 / 4}\left(t^{2} / 2\right) \Gamma(3 / 4)},
$$

where $J_{n}(t)$ is the Bessel function of first kind.

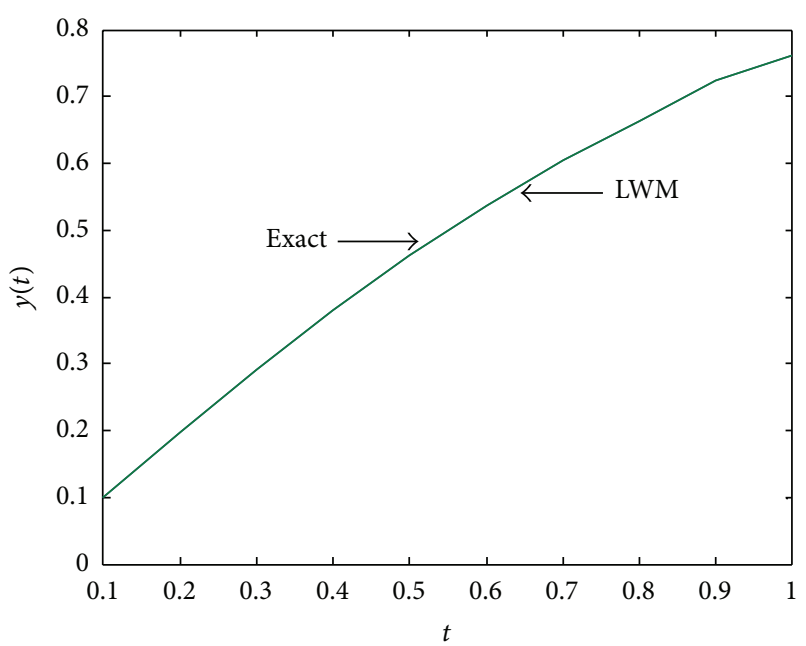

FIGURE 3: Numerical results of Example 2 by LWM for $\alpha=1$.

The integral representation of (69) and (70) is given by

$$
y(t)=1+\frac{2}{\Gamma(\alpha+1)+2 \Gamma(\alpha)} t^{\alpha+2}+I^{\alpha} y^{2}(t)
$$




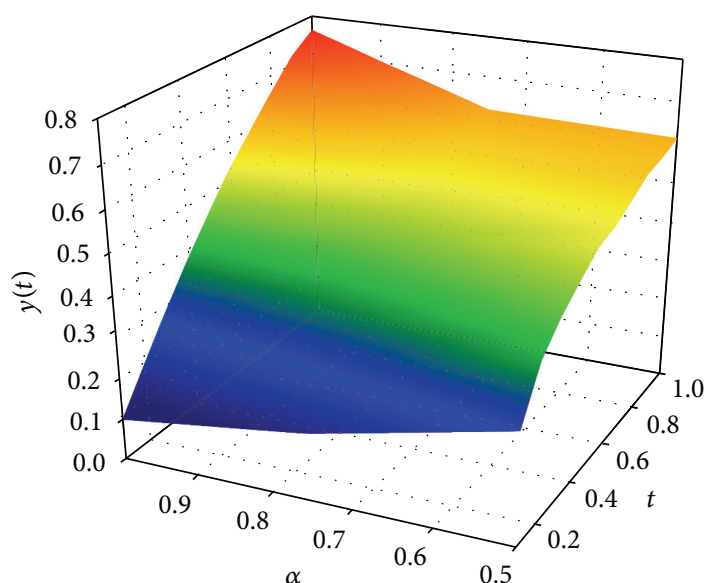

FIGURE 4: Numerical results of Example 2 by LWM for different values of $\alpha$.

Let

$$
y(t)=C^{T} \Psi(t),
$$

and then

$$
\begin{aligned}
I^{\alpha} y(t) & =C^{T} I^{\alpha} \Psi(t) \\
& =C^{T} P_{2^{k-1} M \times 2^{k-1} M}^{\alpha} \Psi(t) .
\end{aligned}
$$

By substituting (73) and (74) into (69), we get the following system of algebraic equations:

$$
\begin{aligned}
C^{T} \Psi(t)= & 1+\frac{2}{\Gamma(\alpha+1)+2 \Gamma(\alpha)}\left(C^{T} \Psi(t)\right)^{\alpha+2} \\
& +C^{T} P_{2^{k-1} M \times 2^{k-1} M}^{2 \alpha} \Psi(t) .
\end{aligned}
$$

By solving the above system of linear equations, we can find the vector $C$. Numerical results are obtained for different values of $k, M$, and $\alpha$. Obtained results for (69) and (70) are shown in Figures 5 and 6. Figure 5 shows the solutions obtained by LWM for different values of $\alpha$ and for $k=2$ and $M=4$. Figure 6 compares the solution obtained by LWM with the exact solution of (69) and (70) when $\alpha=1, k=1$, and $M=2$. So far, there are no published results of absolute error for this problem and hence we are unable to compare absolute error of our method with the existing methods. From these results, we can see that the proposed LWM approach gives the solution which is very close to the exact solution and outperformed recently developed approaches for the nonlinear fractional-order Riccati differential equations in terms of solution quality and convergence criteria.

\section{Conclusions}

Nonlinear fractional-order Riccati differential equations play an important role in the modeling of many biological, physical, chemical, and real life problems. Therefore, it is necessary to develop a method which would give more accurate solutions to such type of problems with greater convergence

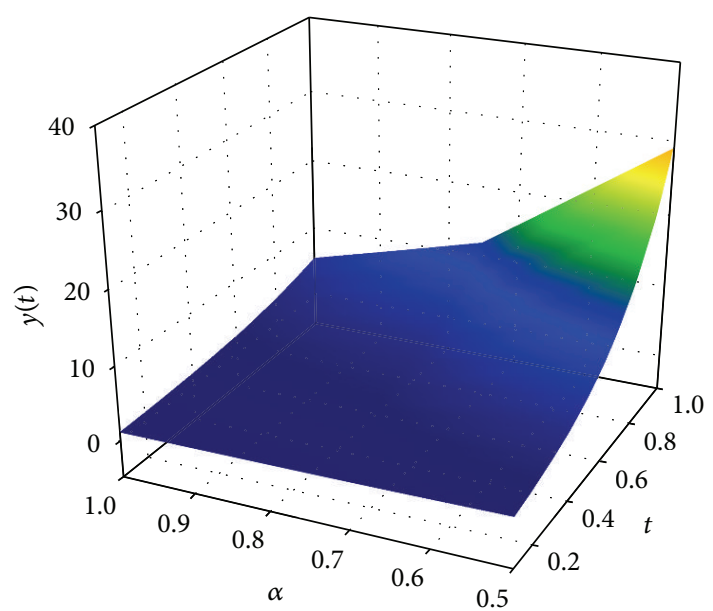

FIgURE 5: Numerical results of Example 3 by LWM for different values of $\alpha$.

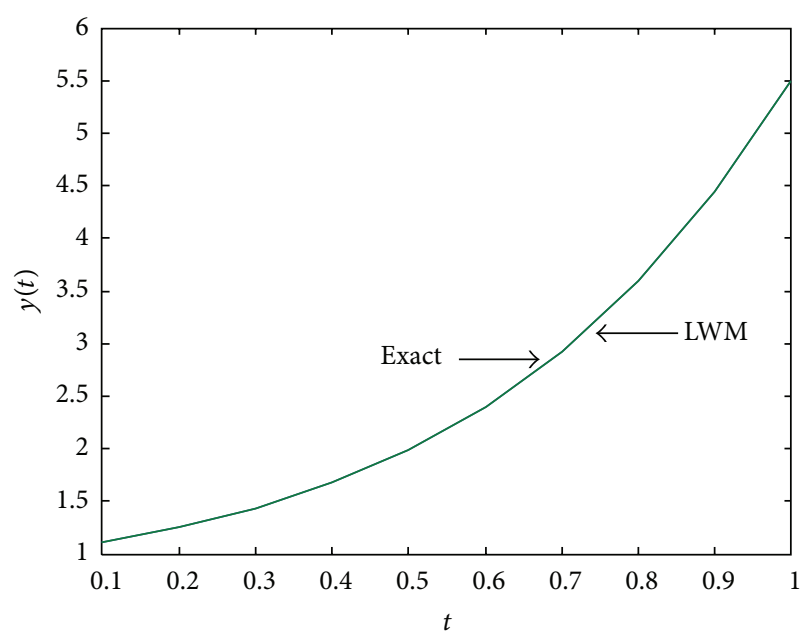

Figure 6: Numerical results of Example 3 by LWM for $\alpha=1$.

criteria. In this work, a Legendre's wavelet operational matrix method called LWM was proposed for solving nonlinear fractional-order Riccati differential equations. Comparison was made for the solutions obtained by the proposed method and with the other recent approaches developed for the same problem through their error analysis; obtained results show that the proposed LWM yields more accurate and reliable solutions even for small values of $M$ and $k$, which assures the best approximate solution in less computational effort. Further, we have discussed the convergence criteria of proposed scheme, which indeed provides the guarantee of consistency and stability of the proposed LWM scheme for the solutions of nonlinear fractional Riccati differential equations.

\section{Conflict of Interests}

The author declares that there is no conflict of interests regarding the publication of this paper. 


\section{References}

[1] N. A. Khan, M. Jamil, A. Ara, and S. Das, "Explicit solution for time-fractional batch reactor system," International Journal of Chemical Reactor Engineering, vol. 9, article A91, 2011.

[2] V. Feliu-Batlle, R. R. Pérez, and L. S. Rodríguez, "Fractional robust control of main irrigation canals with variable dynamic parameters," Control Engineering Practice, vol. 15, no. 6, pp. 673686, 2007.

[3] I. Podlubny, "Fractional-order systems and $P I^{\lambda} D^{\mu}$-controllers," IEEE Transactions on Automatic Control, vol. 44, no. 1, pp. 208214, 1999.

[4] R. Garrappa, "On some explicit Adams multistep methods for fractional differential equations," Journal of Computational and Applied Mathematics, vol. 229, no. 2, pp. 392-399, 2009.

[5] M. Jamil and N. A. Khan, "Slip effects on fractional viscoelastic fluids," International Journal of Differential Equations, vol. 2011, Article ID 193813, 19 pages, 2011.

[6] F. Mohammadi and M. M. Hosseini, "A comparative study of numerical methods for solving quadratic Riccati differential equations," Journal of the Franklin Institute, vol. 348, no. 2, pp. 156-164, 2011.

[7] R. Shankar, Principles of Quantum Mechanics, Plenum Press, New York, NY, USA, 1980.

[8] S. Fraga, M. J. García de la Vega, and E. S. Fraga, The Schrödinger and Riccati Equations, vol. 70 of Lect. Notes. Chem., 1999.

[9] L. B. Burrows and M. Cohen, "Schrodinger's wave equationA lie algebra treatment," in Fundamental World of Quantum Chemistry: A Tribute to the Memory of Per-Olov Löwdin, E. J. Brändas and E. S. Kryachko, Eds., Kluwer, Dordrecht, The Netherlands, 2004.

[10] S. Abbasbandy, "Homotopy perturbation method for quadratic Riccati differential equation and comparison with Adomian's decomposition method," Applied Mathematics and Computation, vol. 172, no. 1, pp. 485-490, 2006.

[11] Z. Odibat and S. Momani, "Modified homotopy perturbation method: application to quadratic Riccati differential equation of fractional order," Chaos, Solitons \& Fractals, vol. 36, no. 1, pp. 167-174, 2008.

[12] N. A. Khan, A. Ara, and M. Jamil, "An efficient approach for solving the Riccati equation with fractional orders," Computers \& Mathematics with Applications, vol. 61, no. 9, pp. 2683-2689, 2011.

[13] H. Aminikhah and M. Hemmatnezhad, "An efficient method for quadratic Riccati differential equation," Communications in Nonlinear Science and Numerical Simulation, vol. 15, no. 4, pp. 835-839, 2010.

[14] S. Abbasbandy, "Iterated He's homotopy perturbation method for quadratic Riccati differential equation," Applied Mathematics and Computation, vol. 175, no. 1, pp. 581-589, 2006.

[15] J. Cang, Y. Tan, H. Xu, and S. Liao, "Series solutions of non-linear Riccati differential equations with fractional order," Chaos, Solitons and Fractals, vol. 40, no. 1, pp. 1-9, 2009.

[16] Y. Tan and S. Abbasbandy, "Homotopy analysis method for quadratic Riccati differential equation," Communications in Nonlinear Science and Numerical Simulation, vol. 13, no. 3, pp. 539-546, 2008.

[17] M. Gülsu and M. Sezer, "On the solution of the Riccati equation by the Taylor matrix method," Applied Mathematics and Computation, vol. 176, no. 2, pp. 414-421, 2006.
[18] Y. Li and L. Hu, "Solving fractional Riccati differential equations using Haar wavelet," in Proceedings of the 3rd International Conference on Information and Computing (ICIC '10), pp. 314317, Wuxi, China, June 2010.

[19] N. A. Khan and A. Ara, "Fractional-order Riccati differential equation: analytical approximation and numerical results," Advances in Difference Equations, vol. 2013, article 185, 2013.

[20] M. A. Z. Raja, J. A. Khan, and I. M. Qureshi, "A new stochastic approach for solution of Riccati differential equation of fractional order," Annals of Mathematics and Artificial Intelligence, vol. 60, no. 3-4, pp. 229-250, 2010.

[21] M. Merdan, "On the solutions fractional Riccati differential equation with modified RIEmann-Liouville derivative," International Journal of Differential Equations, vol. 2012, Article ID 346089, 17 pages, 2012.

[22] N. H. Sweilam, M. M. Khader, and A. M. S. Mahdy, "Numerical studies for solving fractional Riccati differential equation," Applications and Applied Mathematics, vol. 7, no. 2, pp. 595-608, 2012.

[23] C. K. Chui, Wavelets: A Mathematical Tool for Signal Processing, SIAM, Philadelphia, Pa, USA, 1997.

[24] G. Beylkin, R. Coifman, and V. Rokhlin, "Fast wavelet transforms and numerical algorithms, I," Communications on Pure and Applied Mathematics, vol. 44, no. 2, pp. 141-183, 1991.

[25] M. ur Rehman and R. Ali Khan, "The Legendre wavelet method for solving fractional differential equations," Communications in Nonlinear Science and Numerical Simulation, vol. 16, no. 11, pp. 4163-4173, 2011.

[26] S. Balaji, "A new approach for solving Duffing equations involving both integral and non-integral forcing terms," Ain Shams Engineering Journal, 2014.

[27] M. Caputo, "Linear models of dissipation whose Q is almost frequency independent II," Geophysical Journal of the Royal Astronomical Society, vol. 13, pp. 529-539, 1967.

[28] A. A. Kilbas, H. M. Srivastava, and J. J. Trujillo, Theory and Applications of Fractional Differential Equations, vol. 204 of North-Holland Mathematics Studies, Elsevier, 2006.

[29] J. S. Gu and W. S. Jiang, "The Haar wavelets operational matrix of integration," International Journal of Systems Science, vol. 27, no. 7, pp. 623-628, 1996.

[30] M. Razzaghi and S. Yousefi, "Legendre wavelets direct method for variational problems," Mathematics and Computers in Simulation, vol. 53, no. 3, pp. 185-192, 2000.

[31] M. Razzaghi and S. Yousefi, "Legendre wavelets method for constrained optimal control problems," Mathematical Methods in the Applied Sciences, vol. 25, no. 7, pp. 529-539, 2002.

[32] A. Kilicman and Z. A. A. Al Zhour, "Kronecker operational matrices for fractional calculus and some applications," Applied Mathematics and Computation, vol. 187, no. 1, pp. 250-265, 2007. 


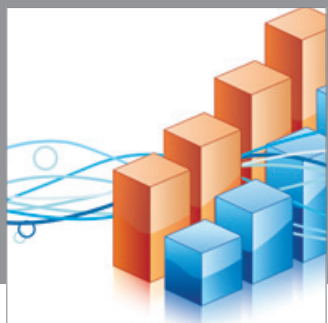

Advances in

Operations Research

mansans

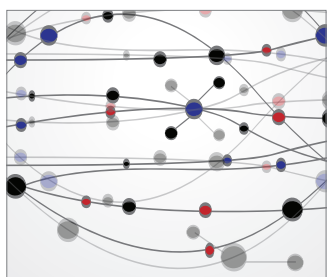

The Scientific World Journal
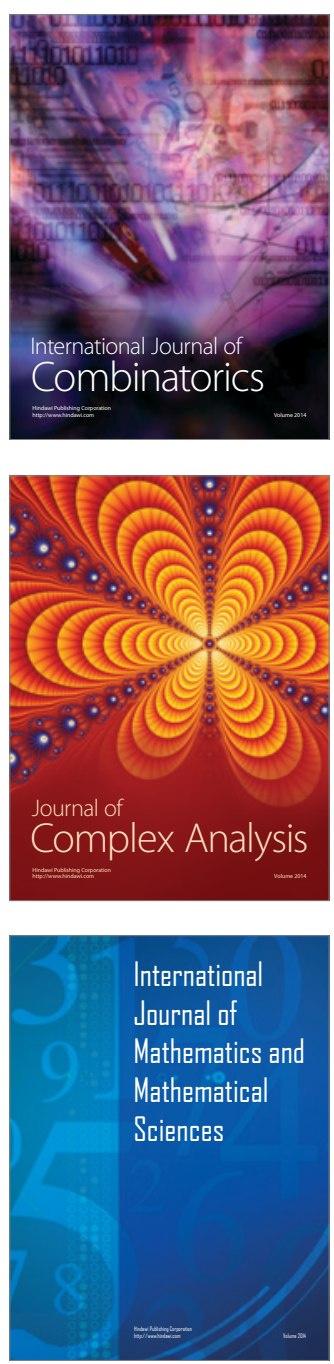
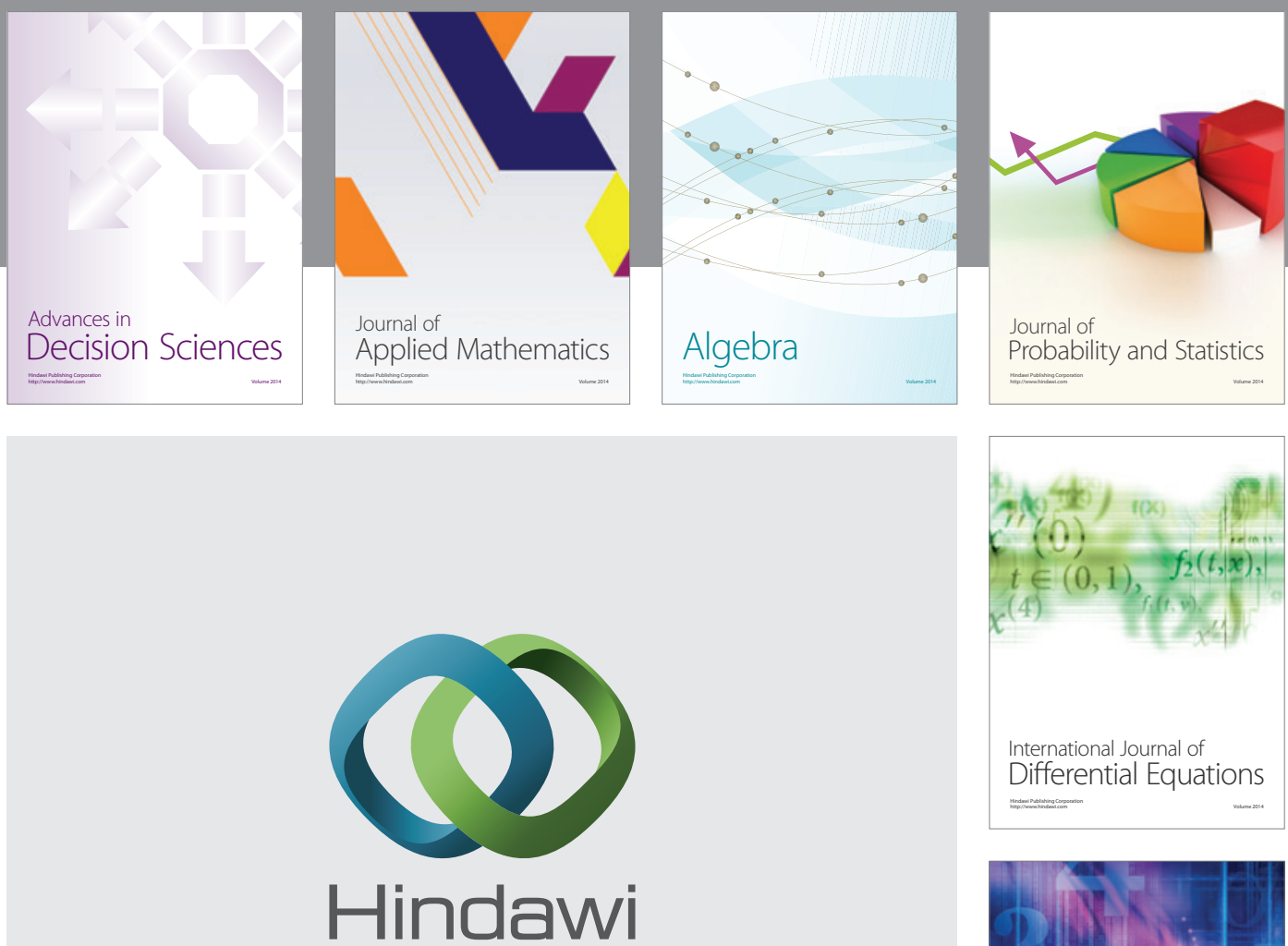

Submit your manuscripts at http://www.hindawi.com
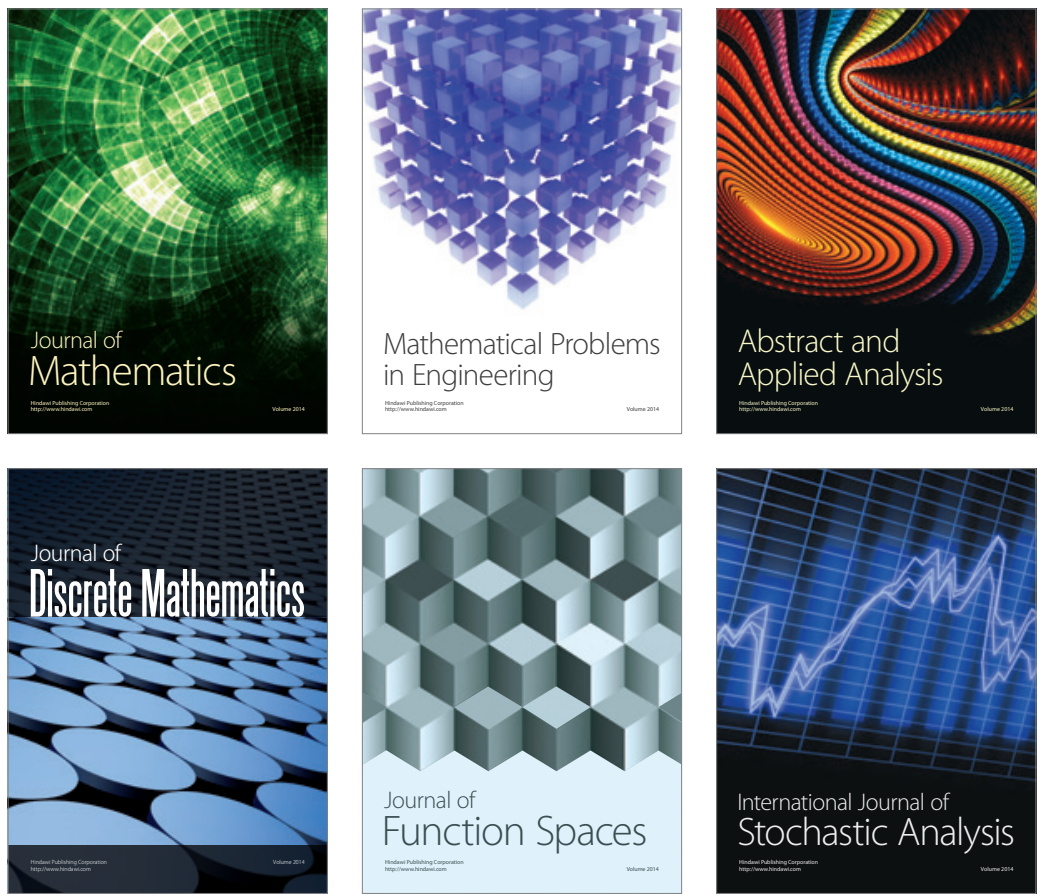

Journal of

Function Spaces

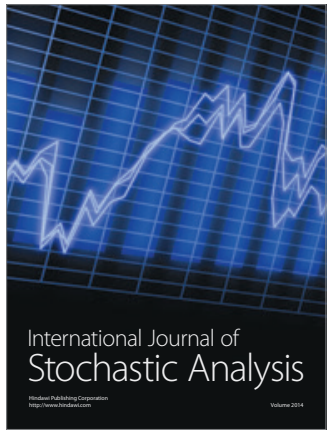

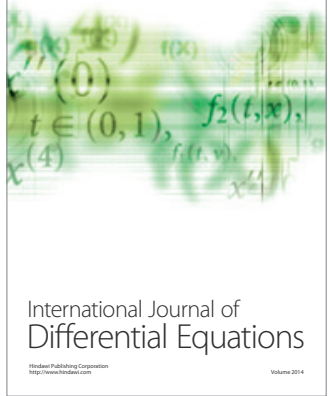
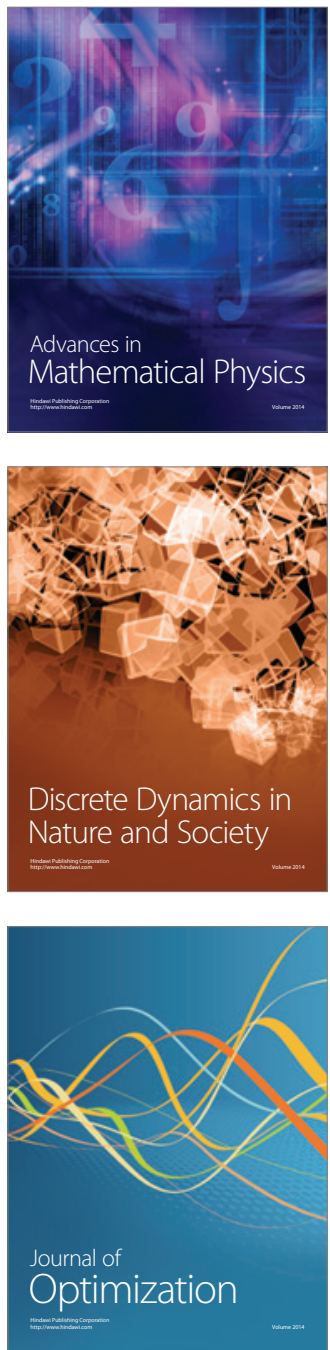